\title{
Spin-transfer torque in magnetic tunnel junctions
}

\author{
Alan Kalitsov, ${ }^{1,2}$ Mairbek Chshiev, ${ }^{3,4}$ Ioannis Theodonis, ${ }^{1,5}$ Nicholas Kioussis,,${ }^{1, *}$ and W. H. Butler ${ }^{3}$ \\ ${ }^{1}$ Department of Physics, California State University, Northridge, California 91330-8268, USA \\ ${ }^{2}$ Institute of Theoretical Physics, University of Kassel, D-34132 Kassel, Germany \\ ${ }^{3}$ MINT Center, University of Alabama, P.O. Box 870209, Tuscaloosa, Alabama, USA \\ ${ }^{4}$ SPINTEC, CEA/CNRS, URA 2512 CEA/CNRS, 38054 Grenoble, France \\ ${ }^{5}$ Department of Physics, National Technical University, GR-15773 Zografou, Athens, Greece \\ (Received 5 December 2008; revised manuscript received 23 February 2009; published 12 May 2009)
}

\begin{abstract}
We present a theoretical study of the spin-transfer torque vector and the tunneling magnetoresistance (TMR) for symmetric magnetic tunnel junctions (MTJ) using the single-band tight-binding model and the nonequilibrium Keldysh formalism. We provide a comprehensive analysis of the effect of band filling and exchange splitting of the FM leads on the bias behavior of the spin-transfer component, $T_{\|}$, in the plane containing the magnetizations of the two magnetic layers, and the fieldlike component, $T_{\perp}$, perpendicular to this plane. We demonstrate that both components of the spin torque and the TMR can exhibit a wide range of interesting and unusual bias behavior. We show that $T_{\|}(V)$ satisfies an expression involving the difference in spin currents between the ferromagnetic (FM) and antiferromagnetic (AF) configurations, which is general and independent of the details of the electronic structure. The spin current for the FM (AF) alignment is shown to have a linear (quadratic) bias dependence, whose origin lies in the symmetric (asymmetric) nature of the barrier. On the other hand, the bias dependence of $T_{\perp}$ is quadratic with $d^{2} T_{\perp} / d V^{2}<0$, and it can change sign at finite bias. Finally, we show that the exchange splitting and band filling have a large effect on the bias dependence of the TMR.
\end{abstract}

DOI: 10.1103/PhysRevB.79.174416 PACS number(s): 85.75.-d, 72.10. $-\mathrm{d}$, 72.25.-b, 73.40.Gk

\section{INTRODUCTION}

In recent years spin-dependent tunneling in magnetic tunnel junctions (MTJ) has become the subject of great interest because of the richness in physical properties it exhibits, and its promising applications in magnetic read heads in computer hard drives, nonvolatile magnetic random access memories (MRAM), and magnetic sensors. ${ }^{1-3}$ The MTJ, consisting of two ferromagnetic (FM) metal electrodes separated by a thin nonmagnetic insulating barrier (B), exhibit the phenomenon of tunneling magnetoresistance (TMR), where the electrical resistance and, hence, the tunneling current depend strongly on the relative orientation of the magnetizations of the electrodes, which can change by a relatively low applied magnetic field. The TMR ratio is defined as, TMR $\equiv \frac{\left(I_{\mathrm{FM}}-I_{\mathrm{AF}}\right)}{I_{\mathrm{AF}}}$, where $I_{\mathrm{FM}}$ and $I_{\mathrm{AF}}$ are the junction currents in the $\mathrm{FM}$ and antiferromagnetic (AF) configurations, respectively, where $I_{\mathrm{AF}}<I_{\mathrm{FM}}$. Recent experiments ${ }^{4-6}$ have shown that the $\mathrm{TMR}$ in crystalline $\mathrm{Fe} / \mathrm{MgO} / \mathrm{Fe}$ tunnel junctions has roomtemperature values that can exceed several hundred percent, as theoretically predicted. ${ }^{7,8}$ Experimental results, which consistently show a decrease in the TMR as a function of bias, are controversial and no consensus has been reached on the physics behind them. ${ }^{9-14}$ Several mechanisms have been proposed to explain this behavior, including inelastic spinflip scattering processes by interfacial magnon excitations, ${ }^{15,16}$ spin-independent two-step elastic tunneling via defect states in the insulating barrier, ${ }^{17}$ voltage-dependent density of states at the Fermi level, ${ }^{18}$ and the electronic structure of the FM electrodes. ${ }^{12,13,19,20}$

While in MTJ structures the relative orientation of magnetizations affects the flow of spin-polarized current, Slonczewski ${ }^{21}$ and Berger ${ }^{22}$ independently predicted a re- verse effect. Namely, the flow of spin-polarized current in noncollinear MTJ can transfer spin angular momentum from the carriers to the ferromagnet and alter the orientation of the corresponding magnetization at sufficiently high current density, even in the absence of an applied field. This phenomenon, known as spin-transfer torque, has since been extensively studied both theoretically ${ }^{23-29}$ and experimentally. ${ }^{30-35}$ A demonstration of the spin-transfer phenomenon is the current-induced magnetic switching (CIMS), which has now been confirmed in numerous experiments in MTJs. ${ }^{36,37}$ Thus, CIMS provides a powerful new tool for the study of spin transport in magnetic nanostructures. Moreover spin-transfer torque can drive nanoscale microwave oscillators. ${ }^{38-40}$

While the presence of the spin torque has been unambiguously observed, its quantitative behavior in a MTJ, especially its bias dependence, has not been understood in detail and remains controversial. ${ }^{32-35}$ The spin torque can be decomposed into a fieldlike component, $T_{\perp}$, and a spin-transfer component, $T_{\|}$, both orthogonal to the magnetization of the free FM, where the first (latter) are perpendicular (parallel) to the plane of the magnetizations of the left and right FM leads, but with different bias behavior. While experiments indicate $^{31-35}$ that the sign of the spin-transfer component, $T_{\|}$, reverses sign on changing the current direction, we have recently predicted an anomalous bias behavior, ${ }^{24}$ where $T_{\|}$can exhibit a sign reversal without a corresponding sign reversal of the bias or even a quadratic bias dependence. Thus, there is pressing need in understanding the origin in the electronic structure responsible for the bias behavior of the spintransfer torque and its relation to TMR, in order to pave the way for new technological applications. ${ }^{41}$

In this work, we present a comprehensive study of the bias behavior of the spin-transfer torque and the TMR, using the one-band tight-binding (TB) model and the nonequilib- 
rium Keldysh formalism. The purpose is to give an extended discussion of the theory presented in Ref. 24, where we presented a brief summary of our results. Here, we provide a detailed investigation of the effect of band filling and exchange splitting of the FM leads on the bias behavior of $T_{\|}$, $T_{\perp}$, and TMR. We demonstrate that the spin-transfer torque can exhibit a wide range of interesting and unusual bias behavior, which can be understood in terms of the dependence of the spin and charge currents on the interplay between the evanescent states of the insulator and the Fermi surfaces of the FM electrodes. On the other hand, we find that the bias dependence of $T_{\perp}(V)$ is quadratic with $d^{2} T_{\perp} / d V^{2}<0$. Interestingly, we find that, depending on band filling and the exchange coupling, $T_{\perp}$ can change sign at finite bias. This may have important practical implications since it suggests that the interlayer exchange coupling (IEC) in MTJ may be tuned with bias. ${ }^{42,43}$ Finally, we show that the TB parameters may have a large effect on the bias dependence of the TMR.

This paper is organized as follows: Section II describes the method used to model the MTJ and to calculate the spintransfer torques. Numerical results for the bias dependence of $T_{\|}, T_{\perp}$, and TMR are presented in Secs. III A-III C, respectively. Finally, conclusions are summarized in Sec. IV.

\section{METHODOLOGY}

The MTJ, shown in Fig. 1, consists of a left and right semi-infinite noncollinear FM leads, separated by a nonmagnetic insulating spacer containing $N$ atomic layers. The magnetization, $\mathbf{M}^{\prime}$, of the right FM lead is along the $z$ axis of the coordinate system shown in Fig. 1. The magnetization, $\mathbf{M}$, of the left FM lead lies in the $x-z$ interfacial plane, i.e., it is rotated by the angle $\theta$ around the axis $y$ (normal to the FM/B interfaces) with respect to $\mathbf{M}^{\prime}$.

The Hamiltonian for the MTJ system is described by the single orbital simple-cubic TB Hamiltonian, which is the sum of the Hamiltonian of the isolated left $(\mathrm{L})$ and right $(\mathrm{R})$ leads, the barrier (B), and the interaction between the left and right leads with the barrier. Namely,

$$
H=H_{L}+H_{R}+H_{B}+H_{\text {int }},
$$

where

$$
\begin{gathered}
H_{L}=\sum_{\sigma ; \lambda} \epsilon_{\lambda}^{\sigma} c_{\lambda}^{\sigma_{\lambda}^{\dagger}} c_{\lambda}^{\sigma}+\sum_{\sigma ; \lambda, \mu} t_{\lambda \mu}^{\sigma} c_{\lambda}^{\sigma^{\dagger} \dagger} c_{\mu}^{\sigma}, \\
H_{R}=\sum_{\sigma ; \lambda^{\prime}} \epsilon_{\lambda^{\prime}}^{\sigma} c_{\lambda}^{\sigma_{\lambda}^{\dagger}} c_{\lambda^{\prime}}^{\sigma}+\sum_{\sigma ; \lambda^{\prime}, \mu^{\prime}} t_{\lambda^{\prime} \mu^{\prime}}^{\sigma} c_{\lambda^{\prime}}^{\sigma^{\dagger}} c_{\mu^{\prime}}^{\sigma}, \\
H_{B}=\sum_{i} \epsilon_{i} c_{i}^{\dagger} c_{i}+\sum_{i, j} t_{i j} c_{i}^{\dagger} c_{j},
\end{gathered}
$$

and

$$
H_{\text {int }}=\sum_{\sigma}\left(t_{a \alpha}^{\sigma} c_{a}^{\sigma \dagger} c_{\alpha}^{\sigma}+t_{b \alpha^{\prime}}^{\sigma} c_{b}^{\sigma \dagger} c_{\alpha^{\prime}}^{\sigma}+h c\right) .
$$

Here, the Greek primed (unprimed) subscripts denote atomic sites in the right (left) FM leads in Fig. 1, respectively, the Latin subscripts denote the sites in the nonmagnetic B
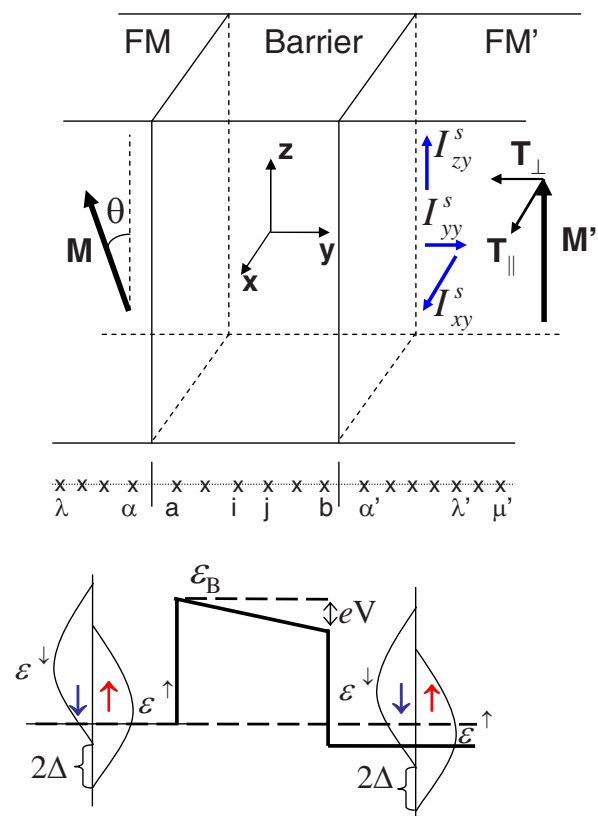

FIG. 1. (Color online) (top) Schematic structure of the MTJ, consisting of left and right semi-infinite FM leads separated by a thin nonmagnetic insulating barrier containing $N$ atomic layers. The magnetization $\mathbf{M}^{\prime}$ of the right $\mathrm{FM}$ lead is along $z$, whereas the magnetization $\mathbf{M}$ of the left lead is rotated by an angle $\theta$ around the $y$ axis with respect to $\mathbf{M}^{\prime}$. The Greek primed (unprimed) letters denote atomic sites in the right (left) FM leads, respectively, and the Latin letters denote the sites in the barrier. (bottom) Schematic illustration of the potential profile, where the spin-resolved density of states in the FM leads have an exchange spin splitting of $2 \Delta$. The $\epsilon^{\uparrow}, \epsilon^{\downarrow}$, and $\epsilon_{B}$ are the on-site energy levels of the majority and minority bands, and the barrier, respectively. The lower dashed line indicates the Fermi level in equilibrium.

spacer, the last (first) site in the left (right) lead next to the left (right) interfaces are denoted by $\alpha\left(\alpha^{\prime}\right)$, while the first (last) site in $B$ are denoted by $a(b) . c_{\mu}^{\sigma}\left(c_{\mu}^{\sigma \dagger}\right)$ annihilates (creates) an electron on site $\mu$ with spin $\sigma, \epsilon_{\lambda}^{\sigma}\left(\epsilon_{i}\right)$ are the spindependent (independent) on-site energies in the lead (barrier), and $t_{\lambda \mu}^{\sigma}\left(t_{i j}\right)$ is the spin-dependent (independent) hopping matrix element between sites $\lambda(i)$ and $\mu(j)$ in the lead (B).

The treatment of the charge- and spin-transport properties of the MTJ is based on an extension of the Keldysh formalism ${ }^{44}$ to the case of noncollinear magnetization between the left and right FM leads, which can be described using $2 \times 2$ matrices in spin space. The one-electron Schrödinger equation for the spin-dependent retarded Green's function, $g_{p q}^{\sigma \sigma^{\prime}}$, for each uncoupled region $\Omega=L, R$ and $B$, can be written as

$$
\begin{aligned}
& \sum_{p_{1}}\left\{\left[\left(E-\varepsilon_{\mathbf{k}_{\mid}}\right) \delta_{p p_{1}}-\bar{H}_{p p_{1}}\right] \hat{I}-\delta H_{p p_{1}}\right. \\
& \left.\times\left(\begin{array}{cc}
\cos \theta & \sin \theta \\
\sin \theta & -\cos \theta
\end{array}\right)\right\}\left(\begin{array}{cc}
g_{p_{1} q}^{\uparrow \uparrow} & g_{p_{1} q}^{\uparrow \downarrow} \\
g_{p_{1} q}^{\downarrow \uparrow} & g_{p_{1} q}^{\downarrow \downarrow}
\end{array}\right)=\delta_{p q} \hat{I},
\end{aligned}
$$

where $p$ and $q$ denote atomic sites in the uncoupled region 
$\Omega, \varepsilon_{\mathbf{k}_{\|}}$is the energy of the in-plane wave vector, $\mathbf{k}_{\|}$, of the Bloch state, and $\hat{I}$ is the $2 \times 2$ unit matrix. The quantities

$$
\bar{H}_{p q}=\varepsilon_{\Omega}^{0} \delta_{p q}+t_{\Omega}^{0}\left(\delta_{p, q+1}+\delta_{p, q-1}\right)
$$

and

$$
\delta H_{p q}=\Delta_{\Omega} \delta_{p q}+\Delta_{\Omega}^{t}\left(\delta_{p, q+1}+\delta_{p, q-1}\right)
$$

describe the spin-averaged and the spin-split parts of the Hamiltonian, respectively, where, $\varepsilon_{\Omega}^{0}=\left(\varepsilon_{\Omega}^{\uparrow}+\varepsilon_{\Omega}^{\downarrow}\right) / 2$, $t_{\Omega}^{0}=\left(t_{\Omega}^{\uparrow}\right.$ $\left.+t_{\Omega}^{\downarrow}\right) / 2, \Delta_{\Omega}=\left(\varepsilon_{\Omega}^{\downarrow}-\varepsilon_{\Omega}^{\uparrow}\right) / 2$, and $\Delta_{\Omega}^{t}=\left(t_{\Omega}^{\uparrow}-t_{\Omega}^{\downarrow}\right) / 2$.

In order to reduce the set of TB parameters, we consider only nearest-neighbor (NN) spin-independent off-diagonal hopping matrix elements, which are the same for both FM leads, the barrier, and the FM/B interfaces, i.e., $t_{\Omega}^{\sigma}=t_{a \alpha}^{\sigma}$ $=t_{b \alpha^{\prime}}^{\sigma}=t$. Furthermore, we consider identical FM leads (symmetric MTJ) with $\Delta_{\mathrm{L}(\mathrm{R})}=\left(\varepsilon_{\mathrm{L}(\mathrm{R})}^{\downarrow}-\varepsilon_{\mathrm{L}(\mathrm{R})}^{\uparrow}\right) / 2$, and $\Delta_{\Omega}^{t}=\Delta_{B}=0$. Under applied bias, $\varepsilon_{\mathrm{R}}^{\sigma}-\varepsilon_{\mathrm{L}}^{\sigma}=\mathrm{eV}$, and the potential inside the insulator, $\varepsilon_{B, n}=\varepsilon_{B}-\mathrm{eV} \frac{n-1}{N-1}$ varies linearly with the layer number $n$.

The retarded $2 \times 2$ Green's function, $G_{p q}^{\sigma \sigma^{\prime}}$, of the entire coupled system can be determined by solving a system of coupled Dyson equations of the form

$$
\hat{G}_{p q}=\hat{g}_{p q}+\hat{g}_{p a} \hat{\Sigma}_{a a} \hat{G}_{a q}+\hat{g}_{p b} \hat{\Sigma}_{b b} \hat{G}_{b q},
$$

which take into account the propagation of the electron across the FM-B interfaces. A similar equation can be written for the advanced Green's function, $\hat{\tilde{G}}_{p q}$, with $\hat{g}_{p q}$ being replaced with $\hat{\tilde{g}}_{p q}$. Here, $\hat{g}$ is the $2 \times 2$ propagator of each uncoupled region determined from Eq. (6), and the self energies at sites $a$ and $b$ are

$$
\hat{\Sigma}_{a a}=t \hat{g}_{\alpha \alpha} t, \quad \hat{\Sigma}_{b b}=t \hat{g}_{\alpha^{\prime} \alpha^{\prime}} t .
$$

Solution of the system of coupled Dyson Eqs. (9) yields analytical expressions for $\hat{G}_{p q}$. For example, the spin diagonal and off-diagonal matrix elements of the advanced Green's function inside the barrier are

$$
G_{a b}^{\uparrow \uparrow}=\frac{g_{a b}}{D^{\uparrow}\left[1-P\left(\sum_{a a}^{\downarrow \uparrow}\right)^{2} / D^{\uparrow} D^{\downarrow}\right]}
$$

and

$$
G_{a b}^{\downarrow \uparrow}=\frac{g_{a b} \Sigma_{a a}^{\downarrow \uparrow}\left[\left(1-g_{b b} \Sigma_{b b}^{\uparrow \uparrow}\right) g_{a a}+g_{a b} g_{b a} \Sigma_{b b}^{\uparrow \uparrow}\right]}{D^{\uparrow} D^{\downarrow}-P\left(\Sigma_{a a}^{\downarrow \uparrow}\right)^{2}} .
$$

Here,

$$
D^{\uparrow(\downarrow)}=\left(1-g_{a a} \Sigma_{a a}^{\uparrow \uparrow(\downarrow \downarrow)}\right)\left(1-g_{b b} \Sigma_{b b}^{\uparrow \uparrow(\downarrow \downarrow)}\right)-g_{a b} g_{b a} \Sigma_{a a}^{\uparrow \uparrow(\downarrow \downarrow)} \Sigma_{b b}^{\uparrow \uparrow(\downarrow \downarrow)}
$$

and

$$
\begin{aligned}
P= & {\left[g_{a b} g_{b a} \Sigma_{b b}^{\uparrow \uparrow}+g_{a a}\left(1-g_{b b} \Sigma_{b b}^{\uparrow \uparrow}\right)\right] } \\
& \times\left[g_{a b} g_{b a} \Sigma_{b b}^{\downarrow \downarrow}+g_{a a}\left(1-g_{b b} \Sigma_{b b}^{\downarrow \downarrow}\right)\right] .
\end{aligned}
$$

Note, that $g_{a b}$ is spin independent because the barrier is nonmagnetic. The expressions for $G_{a b}^{\downarrow \downarrow}$ and $G_{a b}^{\uparrow \downarrow}$ are similar to those above, where $\Sigma \uparrow \uparrow$ and $\Sigma \downarrow \uparrow$ are replaced with $\Sigma \downarrow \downarrow$ and $\Sigma \uparrow \downarrow$.

The kinetic equation for the nonequilibrium $2 \times 2$ Green's function matrix, derived from the Keldysh formalism, ${ }^{45}$ is

$$
\hat{F}_{p q}=\hat{f}_{p q}+\hat{g}_{p q_{1}} \hat{\Sigma}_{q_{1} q_{2}} \hat{F}_{q_{2} q}+\hat{f}_{p q_{1}} \hat{\Sigma}_{q_{1} q_{2}} \hat{\tilde{G}}_{q_{2} q},
$$

where $\hat{f}$ is the nonequilibrium $2 \times 2$ Green's function matrix for the uncoupled leads and the barrier, and the self-energy is

$$
\hat{\Sigma}_{q_{1} q_{2}}=t \hat{I}\left(\delta_{q_{1} \alpha} \delta_{q_{2} a}+\delta_{q_{1} \alpha^{\prime}} \delta_{q_{2} b}\right)+h c .
$$

This form of self-energy arises because the perturbation of the barrier/leads interaction (5) is instantaneous. ${ }^{44}$

Since we are interested in evaluating the spin torque on the right lead we need to determine $F_{\lambda^{\prime} \mu^{\prime}}$, where sites $\lambda^{\prime}$ and $\mu^{\prime} \in \mathrm{R}$. Thus, the above equation becomes ${ }^{44}$

$$
\hat{F}_{\lambda^{\prime} \mu^{\prime}}=\hat{f}_{\lambda^{\prime} \mu^{\prime}}+\hat{g}_{\lambda^{\prime} \alpha^{\prime}} t \hat{F}_{b \mu^{\prime}}+\hat{f}_{\lambda^{\prime} \alpha^{\prime}} t \hat{\widetilde{G}}_{b \mu^{\prime}}
$$

Because the various $\hat{F}$ 's are coupled, one needs to write similar kinetic equations for $\hat{F}_{b \mu^{\prime}}, \hat{F}_{\alpha \mu^{\prime}}, \hat{F}_{\alpha^{\prime} \mu^{\prime}}$, and $\hat{F}_{a \mu^{\prime}}$, which are of the form

$$
\begin{gathered}
\hat{F}_{b \mu^{\prime}}=\hat{g}_{b a} t \hat{F}_{\alpha \mu^{\prime}}+\hat{g}_{b b} t \hat{F}_{\alpha^{\prime} \mu^{\prime}}+\hat{f}_{b a} t \hat{\widetilde{G}}_{\alpha \mu^{\prime}}+\hat{f}_{b b} t \hat{\widetilde{G}}_{\alpha^{\prime} \mu^{\prime}}, \\
\hat{F}_{\alpha \mu^{\prime}}=\hat{g}_{\alpha \alpha} t \hat{F}_{a \mu^{\prime}}+\hat{f}_{\alpha \alpha} t \hat{\widetilde{G}}_{a \mu^{\prime}}, \\
\hat{F}_{\alpha^{\prime} \mu^{\prime}}=\hat{f}_{\alpha^{\prime} \mu^{\prime}}+\hat{g}_{\alpha^{\prime} \alpha^{\prime}} t \hat{F}_{b \mu^{\prime}}+\hat{f}_{\alpha^{\prime} \alpha^{\prime}} t \hat{\widetilde{G}}_{b \mu^{\prime}},
\end{gathered}
$$

and

$$
\hat{F}_{a \mu^{\prime}}=\hat{g}_{a a} t \hat{F}_{\alpha \mu^{\prime}}+\hat{g}_{a b} t \hat{F}_{\alpha^{\prime} \mu^{\prime}}+\hat{f}_{a a} t \hat{\widetilde{G}}_{\alpha \mu^{\prime}}+\hat{f}_{a b} t \hat{\widetilde{G}}_{\alpha^{\prime} \mu^{\prime}}
$$

The vanishing of the density of states of the uncoupled barrier within the energy gap, where the electron-tunneling process occurs, implies that the Green's functions inside the barrier are real,

$$
\hat{g}_{p q}=\hat{\tilde{g}}_{p q}=\hat{g}_{p q} \quad p, q \in B,
$$

and $\hat{f}_{p q}=0$ when $p, q \in B$. The nonequilibrium Green's function matrix for the uncoupled left and right leads at local equilibrium are

$$
\hat{f}_{\lambda \mu}=\left(1-2 f_{L}\right)\left(\hat{g}_{\lambda \mu}-\hat{\widetilde{g}}_{\lambda \mu}\right),
$$

and

$$
\hat{f}_{\lambda^{\prime} \mu^{\prime}}=\left(1-2 f_{R}\right)\left(\hat{g}_{\lambda^{\prime} \mu^{\prime}}-\hat{\widetilde{g}}_{\lambda^{\prime} \mu^{\prime}}\right),
$$

where $f_{L}$ and $f_{R}$ are the Fermi-Dirac distribution function in the isolated left and right leads. Thus, the self-consistent Eqs. (17)-(21) become 


$$
\left\{\begin{array}{l}
\hat{F}_{\lambda^{\prime} \mu^{\prime}}=\hat{f}_{\lambda^{\prime} \mu^{\prime}}+\hat{g}_{\lambda^{\prime} \alpha^{\prime}} t \hat{F}_{b \mu^{\prime}}+\hat{f}_{\lambda^{\prime} \alpha^{\prime}} t \hat{\widetilde{G}}_{b b} t \hat{\widetilde{g}}_{\alpha^{\prime} \mu^{\prime}} \\
\hat{F}_{b \mu^{\prime}}=\hat{g}_{b a} t \hat{F}_{\alpha \mu^{\prime}}+\hat{g}_{b b} t \hat{F}_{\alpha^{\prime} \mu^{\prime}} \\
\hat{F}_{\alpha \mu^{\prime}}=\hat{g}_{\alpha \alpha} t \hat{F}_{a \mu^{\prime}}+\hat{f}_{\alpha \alpha} t \hat{\widetilde{G}}_{a b} t \hat{\widetilde{g}}_{\alpha^{\prime} \mu^{\prime}} \\
\hat{F}_{\alpha^{\prime} \mu^{\prime}}=\hat{f}_{\alpha^{\prime} \mu^{\prime}}+\hat{g}_{\alpha^{\prime} \alpha^{\prime}} t \hat{F}_{b \mu^{\prime}}+\hat{f}_{\alpha^{\prime} \alpha^{\prime}} t \hat{\widetilde{G}}_{b b} t \hat{\tilde{g}}_{\alpha^{\prime} \mu^{\prime}} \\
\hat{F}_{a \mu^{\prime}}=\hat{g}_{a a} t \hat{F}_{\alpha \mu^{\prime}}+\hat{g}_{a b} t \hat{F}_{\alpha^{\prime} \mu^{\prime}} .
\end{array}\right.
$$

Solving the system of linear Eqs. (25) gives

$$
F_{\lambda^{\prime} \mu^{\prime}}=F_{\lambda^{\prime} \mu^{\prime}}^{\text {left }}+F_{\lambda^{\prime} \mu^{\prime}}^{\text {right }},
$$

where

$$
\begin{aligned}
\hat{F}_{\lambda^{\prime} \mu^{\prime}}^{\text {left }}= & \hat{g}_{\lambda^{\prime} \alpha^{\prime}} t(D e n)^{-1} \hat{g}_{b a} t\left[\hat{g}_{\alpha \alpha} t\left(\hat{I}-\hat{g}_{a a} t \hat{g}_{\alpha \alpha} t\right)^{-1} \hat{g}_{a a} t+\hat{I}\right] \\
& \times \hat{f}_{\alpha \alpha} t \hat{\widetilde{G}}_{a b} t \hat{\tilde{g}}_{\alpha^{\prime} \mu^{\prime}}
\end{aligned}
$$

and

$$
\begin{aligned}
\hat{F}_{\lambda^{\prime} \mu^{\prime}}^{\text {right }}= & \hat{f}_{\lambda^{\prime} \mu^{\prime}}+\hat{f}_{\lambda^{\prime} \alpha^{\prime}} t \hat{\widetilde{G}}_{b b} t \hat{\tilde{g}}_{\alpha^{\prime} \mu^{\prime}}+\hat{g}_{\lambda^{\prime} \alpha^{\prime}} t(D e n)^{-1} \\
& \times\left[\hat{g}_{b a} t \hat{g}_{\alpha \alpha} t\left(\hat{I}-\hat{g}_{a a} t \hat{g}_{\alpha \alpha} t\right)^{-1} \hat{g}_{a b}+\hat{g}_{b b}\right] \\
& \times t\left(\hat{f}_{\alpha^{\prime} \mu^{\prime}}+\hat{f}_{\alpha^{\prime} \alpha^{\prime}} t \hat{\widetilde{G}}_{b b} t \hat{\tilde{g}}_{\alpha^{\prime} \mu^{\prime}}\right) .
\end{aligned}
$$

Here,

$$
\text { Den }=\hat{I}-\hat{g}_{b b} t \hat{g}_{\alpha^{\prime} \alpha^{\prime}} t-\hat{g}_{b a} t \hat{g}_{\alpha \alpha} t\left(\hat{I}-\hat{g}_{a a} t \hat{g}_{\alpha \alpha} t\right)^{-1} \hat{g}_{a b} t \hat{g}_{\alpha^{\prime} \alpha^{\prime}} t \text {. }
$$

Having determined the $\hat{F}_{p q}$, the Keldysh Green's function is ${ }^{45}$

$$
\hat{G}_{p q}^{<}=\frac{1}{2}\left[\hat{F}_{p q}+\hat{\widetilde{G}}_{p q}-\hat{G}_{p q}\right] .
$$

Finally, the charge and spin-current density are

$$
\begin{gathered}
I=\frac{e t}{2 \pi \hbar} \int \operatorname{Tr}_{\sigma}\left[\hat{G}_{p+1, p}^{<\sigma, \sigma^{\prime}}-\hat{G}_{p, p+1}^{<\sigma, \sigma^{\prime}}\right] d E d \mathbf{k}_{\|}, \\
\mathbf{I}_{p, p+1}^{s}=\frac{t}{4 \pi} \int \operatorname{Tr}_{\sigma}\left[\left(\hat{G}_{p+1, p}^{<\sigma, \sigma^{\prime}}-\hat{G}_{p, p+1}^{<\sigma, \sigma^{\prime}}\right) \boldsymbol{\sigma}\right] d E d \mathbf{k}_{\|},
\end{gathered}
$$

respectively, where $\boldsymbol{\sigma}=\left(\sigma_{x}, \sigma_{y}, \sigma_{z}\right)$ is a vector of the Pauli matrices.

While in general the spin current is a tensor, ${ }^{46}$ in our case only the $I_{x y}^{s}, I_{y y}^{s}$, and $I_{z y}^{s}$ (see Fig. 1) components are nonzero because the transport is along the $\mathbf{y}$ direction. In contrast to the charge current, $I$, which is conserved across the MTJ, the spin current, $\mathbf{I}^{s}$, is not conserved due to the local exchange field inside the FM leads, ${ }^{46}$ i.e., $\nabla \cdot \mathbf{I}^{s} \neq 0$. Due to conservation of the total angular momentum, the spin current lost at an atomic site is transferred to its local magnetic moment, thereby exerting a local spin-transfer torque ${ }^{46,47} \mathbf{T}_{\lambda^{\prime}}$ on site $\lambda^{\prime}$ in the right FM lead, given by

$$
\mathbf{T}_{\lambda^{\prime}}=-\nabla \cdot \mathbf{I}^{s}=\mathbf{I}_{\lambda^{\prime}-1, \lambda^{\prime}}^{s}-\mathbf{I}_{\lambda^{\prime}, \lambda^{\prime}+1}^{s},
$$

where the second equality represents the discrete form of the divergence of the spin current. The $z$ component of $\mathbf{T}_{\lambda^{\prime}}$ vanishes because $I_{z\left(\lambda^{\prime}, \lambda^{\prime}+1\right)}^{s}=I_{z\left(\lambda^{\prime}-1, \lambda^{\prime}\right)}^{s}=(\hbar / 2 e)\left(I^{\uparrow}-I^{\downarrow}\right)$, where $I^{\uparrow(\downarrow)}$ is the majority (minority) charge current given by the diagonal matrix elements in Eq. (31). Note, the charge and spin-current densities Eqs. (31) and (32) are calculated in $\mathrm{A} / \square$ and $\mathrm{eV} / \square$, where $\square$ denotes the interfacial unit area.

Using Eq. (33), one can determine the net spin-transfer torque on the right FM lead (transverse to the magnetization), as the sum of local torques,

$$
\mathbf{T}=\sum_{\lambda^{\prime}=0}^{\infty}\left(\mathbf{I}_{\lambda^{\prime}-1, \lambda^{\prime}}^{s}-\mathbf{I}_{\lambda^{\prime}, \lambda^{\prime}+1}^{s}\right)=\mathbf{I}_{-1,0}^{s}-\mathbf{I}_{\infty, \infty}^{s}=\mathbf{I}_{-1,0}^{s} .
$$

Here the subscripts -1 and 0 refer to the last site inside the barrier and the first site in the right FM lead, respectively. In the above equation, $\mathbf{I}_{\infty, \infty}^{s}=0$ because the components of $\mathbf{I}_{\lambda^{\prime}, \lambda^{\prime}+1}^{s}$ transverse to $\mathbf{M}^{\prime}$ decay to zero as $\lambda^{\prime} \rightarrow \infty$. ${ }^{48,49}$ Thus, the total spin torque exerted on the right FM lead is simply the spin current at the I/FM interface. ${ }^{46}$ The fieldlike, $T_{\perp}$, and spin-transfer, $T_{\|}$, components of the spin torque on the right FM lead, shown in Fig. 1, are along the $\hat{\mathbf{M}}^{\prime} \times\left(\hat{\mathbf{M}} \times \hat{\mathbf{M}}^{\prime}\right)$ and $\hat{\mathbf{M}} \times \hat{\mathbf{M}}^{\prime}$ directions, respectively, where $\hat{\mathbf{M}}\left(\hat{\mathbf{M}}^{\prime}\right)$ are the unit vectors along the magnetization direction of the left (right) lead, respectively.

\section{RESULTS AND DISCUSSION}

In a recent paper we presented a brief summary of the anomalous bias dependence of the spin torque in MTJ. ${ }^{24}$ Here we provide a fuller account of the results including the bias dependence of the TMR. We vary two model parameters, the spin-averaged on-site energy, $\varepsilon^{0}=\left(\varepsilon^{\uparrow}+\varepsilon^{\downarrow}\right) / 2$, which controls the band filling and the exchange splitting, $\Delta=\left(\varepsilon^{\downarrow}-\varepsilon^{\uparrow}\right) / 2$. The NN hopping matrix element, $t=-1 \mathrm{eV}$, in all regions, $\varepsilon_{B}=9 \mathrm{eV}$, the Fermi energy $E_{F}=0 \mathrm{eV}$, and the number of barrier sites is $N=3$. The choice of the parameters including the hopping matrix element provides a realistic choice for systems based on magnetic transition metals and their alloys. ${ }^{50,51}$ In order to increase the band filling, we believe that different class of magnetic materials should be suggested, for example, alloying $\mathrm{Fe}(\mathrm{Co})$ with impurities which can fill up the majority $\Delta_{1}$ band.

\section{A. Voltage behavior of the spin-transfer torque}

In Fig. 2 we display the bias dependence of the net spintransfer torque on the right lead, $T_{\|}$, for $\theta=\pi / 2$, various values of $\Delta$ and for $\varepsilon^{0}=3,0,-3 \mathrm{eV}$, corresponding to $1 / 4$ (top panel), 1/2 (middle panel), and $3 / 4$ band filling (bottom panel), respectively. For the case of $3 / 4$ filling, $T_{\|}$increases monotonically both with bias and exchange splitting, with $\left(\frac{\partial T_{\|}}{\partial V}\right)_{\Delta}>0$ and $\left(\frac{\partial T_{\|}}{\partial \Delta}\right)_{V}>0$. On the other hand, for the half filled case, while $\left(\frac{\partial T_{\|}}{\partial V}\right)_{\Delta}>0, T_{\|}$increases with $\Delta$ up to the value of about $4 \mathrm{eV}$ and then decreases with further increase in $\Delta$. Finally, for the $1 / 4$ band filling the dependence of $T_{\|}$on both 


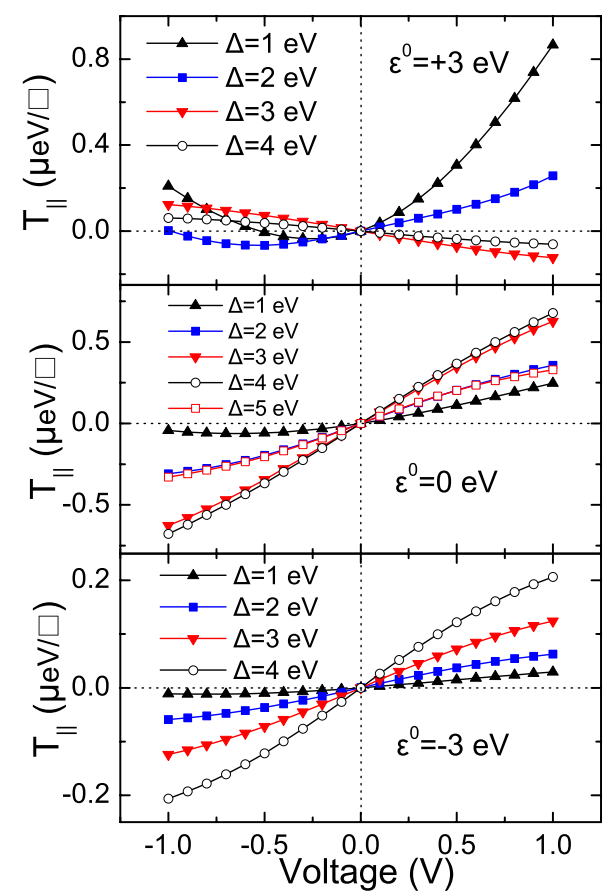

FIG. 2. (Color online) Bias dependence of parallel spin-transfer torque, $T_{\|}$, for $\theta=\pi / 2$, various values of the exchange splitting $\Delta$ and three values of the spin-averaged on-site energy $\varepsilon^{0}$, corresponding to $1 / 4$ (top panel), 1/2 (middle panel), and 3/4 filling (bottom panel), respectively.

bias and exchange splitting is nonmonotonic, with the spintransfer torque reversing sign without a sign reversal of the bias for low values of $\Delta$. Interestingly, for higher $\Delta$ values ( 3 and $4 \mathrm{eV}$ ), the bias dependence renders monotonic with $\left(\frac{\partial T_{\|}}{\partial V}\right)_{\Delta}<0$. Thus, the bias behavior of $T_{\|}$shows a wide range of unusual behavior depending on the band filling and exchange splitting, and is consistent with that observed in recent experiments. ${ }^{32,33,52}$

We would like to point out here that the discrepancies in the sign of the spin-transfer torques in the cited references, as well as in a number of theoretical works, ${ }^{24,26-29}$ are just matters of sign convention, as was also noted in Ref. 27. In addition, the slight bias asymmetry of the in-plane torque in Ref. 33 can be explained by the possible intervention of the minority electrons, which leads to deviation from the "halfmetallic"-like regime for which the variation in the parallel torque is always monotonic (symmetric). This is explained further in the text and is clearly demonstrated in the top panel in Fig. 6 by the curves denoted with open squares and open circles, respectively. These two curves may represent differences in observations reported in Refs. 32 and 33. Also in our previous work, ${ }^{24}$ we did not include the curve corresponding to the half-metallic case, which was obviously symmetric in shape, similar to that reported in the $a b$ initio calculations by Heiliger et al. ${ }^{28}$

The mechanism responsible for the bias behavior of $T_{\|}$ can be understood by employing the generalized equivalent circuit model for MTJ, ${ }^{23,24}$ where the parallel component of the spin-transfer torque on the right lead has the universal form

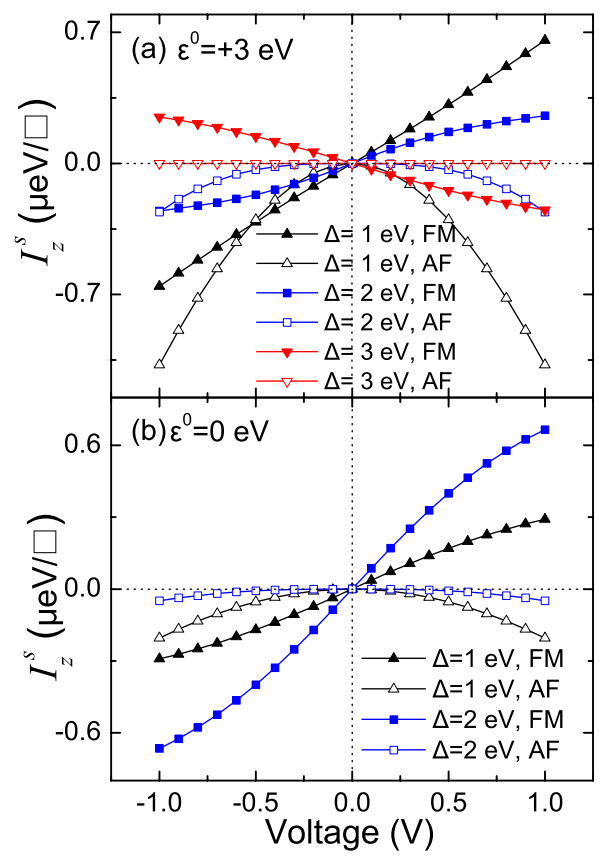

FIG. 3. (Color online) Bias dependence of the spin-current density, $I_{z}^{s}$, for the FM (open symbols) and AF (closed symbols) configurations, respectively, in Eq. (35). $I_{z}^{s}$ is displayed for various values of the exchange interaction $\Delta$ and for $\varepsilon^{0}=+3,0 \mathrm{eV}$, corresponding to the top and middle panels of Fig. 2, respectively.

$$
T_{\|}=\frac{I_{z}^{s}(0)-I_{z}^{s}(\pi)}{2} \hat{\mathbf{M}}^{\prime} \times\left(\hat{\mathbf{M}} \times \hat{\mathbf{M}}^{\prime}\right)
$$

This expression is general and independent of the details of the electronic structure for thick barriers. Our numerical results both for the TB model and the free-electron model (not presented here) confirm the validity of this relation for any parameter set and bias. Here, $I_{z}^{S}(0)=\frac{\hbar}{2 e}\left[I^{\uparrow}(0)-I^{\downarrow}(0)\right]$ and $I_{z}^{S}(\pi)=\frac{\hbar}{2 e}\left[I^{\uparrow}(\pi)-I^{\downarrow}(\pi)\right]$ are the spin-current densities along the direction of $\mathbf{M}^{\prime}$ for the FM and AF configurations, respectively. This result is important because it reduces the calculation of $T_{\|}(\theta)$ simply to the evaluation of spin-current densities for the FM and AF configurations, ${ }^{23,24}$ and is consistent with those in spin valves. ${ }^{48}$

In order to elucidate the underlying mechanism responsible for the bias dependence of $T_{\|}$, we display in Fig. 3 the bias dependence of $I_{z}^{S}(0)$ and $I_{z}^{S}(\pi)$ for the FM (solid symbols) and AF (open symbols) orientations, respectively, for various values of the exchange splitting and for $\varepsilon^{0}=$ $+3,0 \mathrm{eV}$, corresponding to the top and middle panels of Fig. 2 , respectively. One can clearly see that $I_{z}^{S}(0)\left[I_{z}^{S}(\pi)\right]$ is an odd (even) function of bias for $-1 V<V<1 V$. We have shown ${ }^{24}$ that this different bias behavior can be understood on the basis of the Brinkman tunnel model ${ }^{53}$ for asymmetric barriers, generalized so as to take into account both spin channels. Namely, in the FM configuration, the majority and minority electrons tunnel through a symmetric barrier but with different barrier heights for each spin channel. On the other hand, in the AF configuration, both spin channels tunnel through asymmetric barriers with the same average barrier height, but with barrier asymmetry of opposite sign. ${ }^{24}$ 


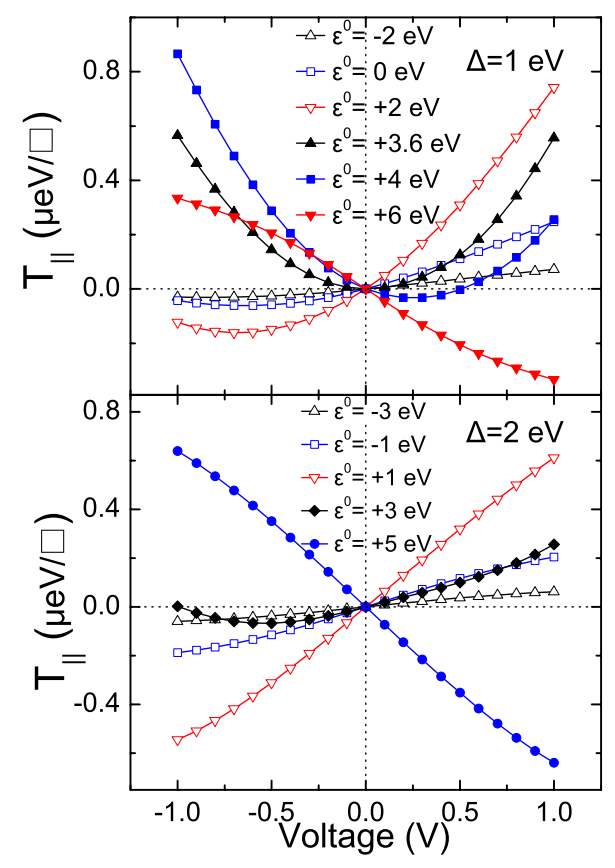

FIG. 4. (Color online) Bias dependence of parallel spin-transfer torque, $T_{\|}$, for $\theta=\pi / 2$, various values of the spin-averaged on-site energy $\varepsilon^{0}$ and for exchange splitting of $\Delta=1 \mathrm{eV}$ (top panel) and $\Delta=2 \mathrm{eV}$ (bottom panel).

Note, that for $\varepsilon^{0}=+3 \mathrm{eV}, \frac{\partial E_{z}^{T}(0)}{\partial V}$ decreases as $\Delta$ increases and reverses sign for $\Delta=3 \mathrm{eV}$. Similarly, $\left|\frac{\partial^{2} E_{2}^{S}(\pi)}{\partial V^{2}}\right|$ decreases with increasing $\Delta$. In the half-metallic case $(\Delta=3 \mathrm{eV}), I_{z}^{S}(\pi)$ vanishes identically for this bias range due to the absence of minority states available for tunneling. Consequently, the odd bias dependence of $T_{\|}$for $\varepsilon^{0}=+3 \mathrm{eV}$ and $\Delta=3 \mathrm{eV}$ arises solely from the bias dependence of $I_{z}^{S}(0)$. On the other hand, $\frac{\partial I_{z}^{(}(0)}{\partial V}$ increases with increasing $\Delta$ for $\varepsilon^{0}=0 \mathrm{eV}$. Thus, $T_{\|}$is an odd function of $\mathrm{V}$ when $I_{z}^{S}(0) \gg I_{z}^{S}(\pi)$ (for example in the half-metallic case), while the nonmonotonic behavior occurs when $I_{z}^{S}(0) \approx I_{z}^{S}(\pi)$. The bias behavior of the spin torque for the $3 / 4$ band filling (not shown here) can be explained similarly in terms of the interplay of the spin currents of the $\mathrm{FM}$ and $\mathrm{AF}$ configurations, respectively.

These results raise the interesting question whether $T_{\|}$can be an even function of bias in the opposite limit where $I_{z}^{S}(0) \ll I_{z}^{S}(\pi)$. In Fig. 4 we plot the bias dependence of $T_{\|}$for $\theta=\pi / 2$ and fixed value of exchange splitting, but different values of the spin-averaged on-site energy $\varepsilon^{0}$ corresponding to different band filling. Namely, both the majority and minority bands are rigidly shifted, but with $\Delta$ kept fixed to $1 \mathrm{eV}$ $(2 \mathrm{eV})$ in the top (bottom) panel. The bias dependence of the corresponding longitudinal spin currents for the FM and AF orientations, respectively, is shown in Fig. 5, for the same values of $\Delta$ and $\varepsilon^{0}$ used in Fig. 4 . One can note that for $\Delta$ $=1 \mathrm{eV}$ upon increasing $\varepsilon^{0}$ (decreasing band filling) the bias dependence of $T_{\|}$changes from monotonic $\left(\varepsilon^{0}=-2,0 \mathrm{eV}\right)$, to nonmonotonic $\left(\varepsilon^{0}=2,4 \mathrm{eV}\right)$, and to purely quadratic $\left(\varepsilon^{0}\right.$ $=3.6 \mathrm{eV}$ ). In the latter case, $I_{z}^{S}(0) \approx 0$ (solid black triangles in top panel of Fig. 5) in this bias range, and $T_{\|}$is solely determined by $I_{z}^{s}(\pi)$. Thus, the interplay between the odd or even bias dependence of $I_{z}^{(s)}(0)$ and $I_{z}^{(s)}(\pi)$, respectively, in

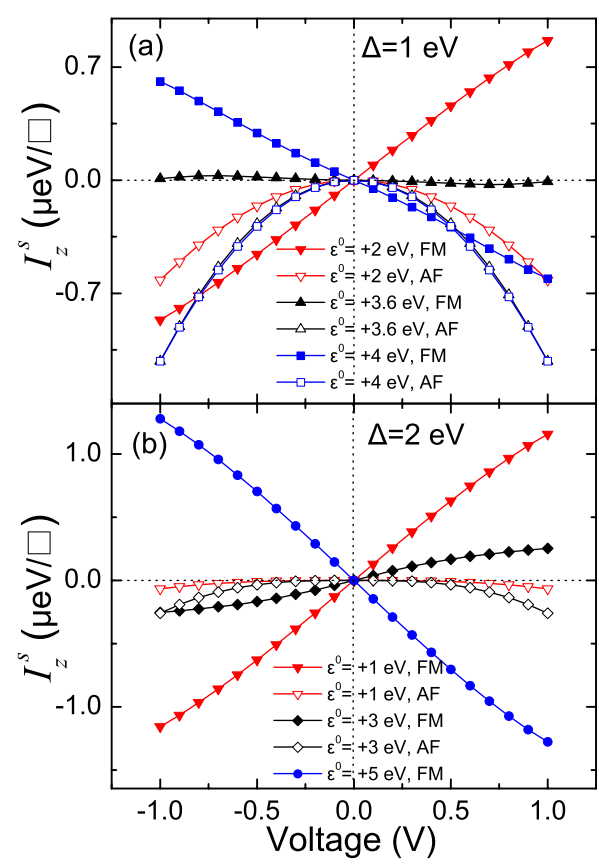

FIG. 5. (Color online) Bias dependence of the longitudinal spincurrent density, $I_{z}^{s}$, in Eq. (35) for FM (closed symbols) and AF (open symbols) configurations, respectively, for various values of $\Delta$ and the spin-averaged on-site energy $\varepsilon^{0}$, corresponding to those in Fig. 2.

Eq. (35) is responsible for the bias dependence of $T_{\|}$in Figs. 2 and 4 . This competition can be selectively tuned by varying $\Delta$ and the band filling $\left(\varepsilon^{0}\right)$, giving rise to a wide range of bias behavior. The monotonic bias dependence of $T_{\|}$for the case of half-filling (middle panel in Fig. 2) is due to the spin current of the FM configuration, $I_{z}^{s}(0)$, which dominates over that of the AF configuration.

Figure 6 displays the bias dependence of $T_{\|}$for $\theta=\pi / 2$, for $\varepsilon^{\uparrow}=+3 \mathrm{eV}$ (top panel), $0 \mathrm{eV}$ (middle panel), and $-3 \mathrm{eV}$ (bottom panel), corresponding to $1 / 4,1 / 2$, and $3 / 4$ band filling, respectively, and for various values of $\varepsilon^{\downarrow}$. In contrast to Figs. 2 and 4, where both the majority and minority bands were rigidly shifted but where either $\varepsilon^{0}$ or $\Delta$ was fixed, in Fig. 6 we examine the effect of the rigid shift of the minority band only, where both $\varepsilon^{0}$ and $\Delta$ change simultaneously. Similarly to Figs. 2 and 4 , the bias behavior of the spintransfer torque exhibits a wide range of behavior, including a purely quadratic one for $\varepsilon^{\uparrow}=+3 \mathrm{eV}$ and $\varepsilon^{\downarrow}=+4.2 \mathrm{eV}$, corresponding to $\varepsilon^{0}=3.6 \mathrm{eV}$ and $\Delta=1.2 \mathrm{eV}$.

Overall, the results indicate that $d I_{z}^{s}(0) / d V>0$ for $\varepsilon^{0}$ $<3.6 \mathrm{eV}$ and $d I_{z}^{S}(0) / d V<0$ for $\varepsilon^{0}>3.6 \mathrm{eV}$, except for the case of low band filling $\left(\varepsilon^{0}=+3 \mathrm{eV}\right.$ ) and $\Delta \geq 3 \mathrm{eV}$ (top panel in Fig. 3), where $d I_{z}^{s}(0) / d V<0$. In order to elucidate the origin in the electronic structure responsible for the bias dependence of the longitudinal spin current, $I_{z}^{S}(0)$, for the FM orientation shown in Figs. 3 and 5, we plot in Fig. 7 the non-spin-polarized current as a function of the spin-averaged on-site energy level, $\varepsilon^{0}$, for several bias values. Interestingly, for all biases the charge current exhibits a maximum at about $\varepsilon_{\max }^{0}=3.6 \mathrm{eV}$, where $I_{z}^{s}(0)$ vanishes also. Since the spinpolarized case can be derived from the non-spin-polarized 


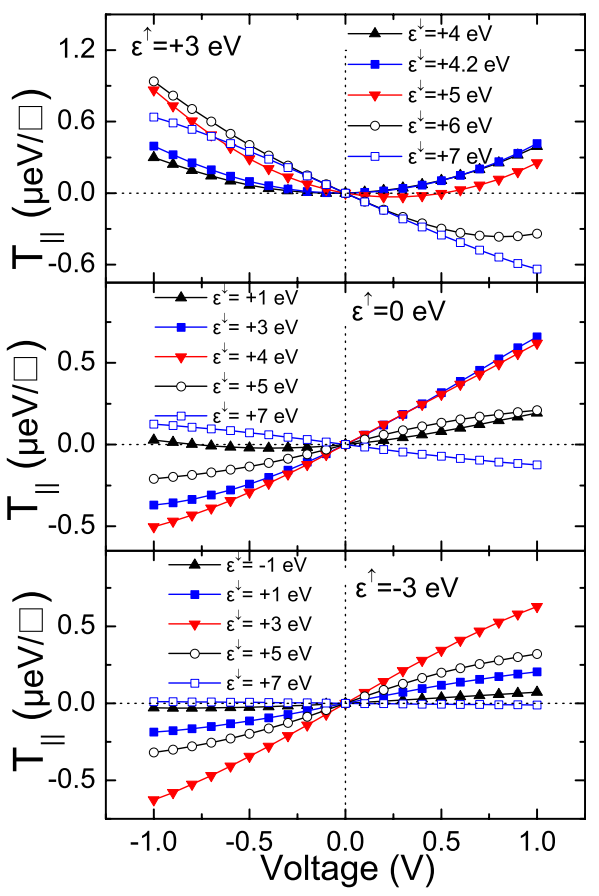

FIG. 6. (Color online) Bias dependence of parallel spin-transfer torque, $T_{\|}$, for $\theta=\pi / 2$, various values of the minority on-site energy $\varepsilon^{\downarrow}$, and for three values of the majority on-site energy $\varepsilon^{\uparrow}$, corresponding to $1 / 4$ (top panel), 1/2 (middle panel), and 3/4 band filling (bottom panel), respectively.

one by adding or subtracting $\Delta$ to $\varepsilon_{\max }^{0}$, i.e., $\varepsilon^{\uparrow(\downarrow)}=\varepsilon_{\max }^{0} \mp \Delta$, the vanishing of $I_{z}^{s}(0)$ results from the fact that $I$ is symmetric about $\varepsilon_{\max }^{0}$, at least for small $\Delta$, yielding $I^{\uparrow}(0)=I^{\downarrow}(0)$. This result is valid up to an exchange-splitting value at which the leads become half-metallic, i.e., $\varepsilon^{0} \mp \Delta>E_{F}+6|t|$. Figure 7 also explains the fact that for all cases with $\varepsilon^{0}<3.6 \mathrm{eV}$ $\left(\varepsilon^{0}>3.6 \mathrm{eV}\right), d I_{z}^{S}(0) / d V>0\left(d I_{z}^{S}(0) / d V<0\right)$.

In order to understand the underlying mechanism responsible for the variation in the charge current, $I$, with $\varepsilon^{0}$, we display in Figs. 8(a)-8(c) the $\mathbf{k}_{\|}$-resolved charge current at $E_{F}=0 \mathrm{eV}$ and at $0.1 \mathrm{~V}$ bias, for three values of $\varepsilon^{0}$ of $-3,0$, and $+3 \mathrm{eV}$, respectively. Here, $\mathbf{k}_{\|}$is the in-plane wave vector in the two-dimensional surface Brillouin zone (BZ). Interestingly, the maximum value of the current for $1 / 4$ filling $\left(\varepsilon^{0}\right.$ $=+3 \mathrm{eV}$ ) occurs in the vicinity of $\mathbf{k}_{\|}=0$, with the maximum value shifting toward the edges $\left(k_{x} \approx k_{z} \approx \pi\right)$ of the $\mathrm{BZ}$ as the

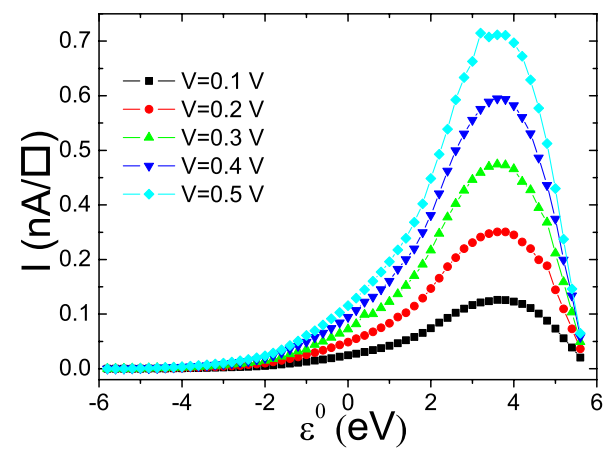

FIG. 7. (Color online) Charge current density versus on-site energy $\varepsilon^{0}$ for several values of applied voltage.

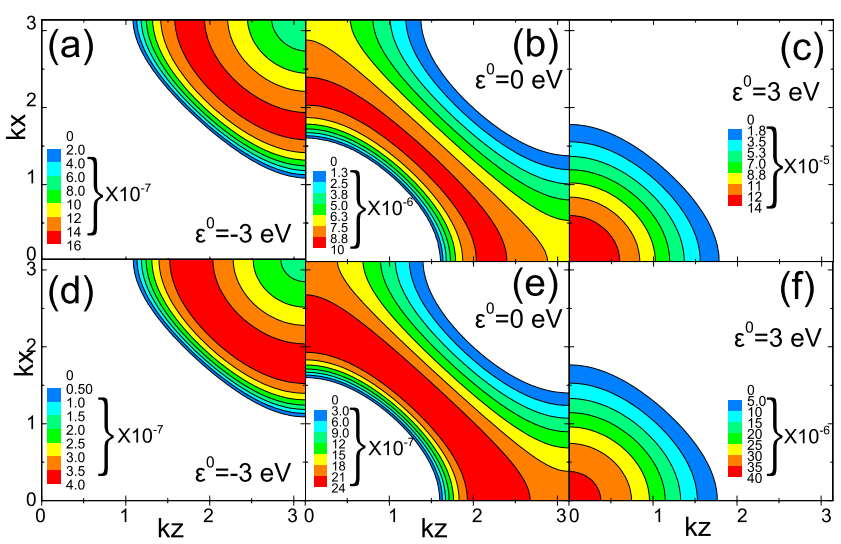

FIG. 8. (Color online) [(a)-(c)]: $\mathbf{k}_{\|}$-resolved current in the twodimensional BZ, calculated from Eq. (31), at $E_{F}=0$, for $0.1 \mathrm{~V}$ bias, and for $\varepsilon^{0}$ of $-3,0$, and $+3 \mathrm{eV}$, respectively. [(d)-(f)]: Corresponding zero-bias $\mathbf{k}_{\|}$-resolved transmission probability, calculated from the simplified Eq. (36), for the same $\varepsilon^{0}$ values.

band filling increases to $3 / 4\left(\varepsilon^{0}=-3 \mathrm{eV}\right)$. For the case of $1 / 2$ filling $\left(\varepsilon^{0}=0 \mathrm{eV}\right)$ the maximum current value occurs at $k_{x}$ $\approx k_{z} \approx \frac{2 \pi}{3}$. It is important to note that the current decreases by about two orders of magnitude as the band filling increases from $1 / 4$ to $3 / 4$.

The variation in the $\mathbf{k}_{\|}$-resolved current at $E_{F}$ with $\varepsilon^{0}$ can be explained by the interplay between the electrode states at the interface and the evanescent states in the barrier. For the simple-cubic TB Hamiltonian in Eq. (1) the zero-bias transmission probability at $E_{F}=0 \mathrm{eV}$ is of the form ${ }^{21,54,55}$

$$
T\left(\mathbf{k}_{\|}\right)=\left[T_{\text {int }}\left(\mathbf{k}_{\|}\right)\right]^{2} e^{-2 N q_{y} a}=\frac{4 \sinh ^{2} q_{y} a \sin ^{2} k_{y} a}{\left(\cosh q_{y} a-\cos k_{y} a\right)^{2}} e^{-2 N q_{y} a},
$$

where

$$
\begin{aligned}
& \cos \left(k_{y} a\right)=-\epsilon^{0} / 2 t-\cos k_{x} a-\cos k_{z} a, \\
& \cosh \left(q_{y} a\right)=-\epsilon_{B} / 2 t-\cos k_{x} a-\cos k_{z} a .
\end{aligned}
$$

Equation (36) represents the TB analog of the generalized Julliére model for free electrons, ${ }^{21,54,55}$ where the tunneling transmission factorizes into the product of two interfacial transmission functions, $T_{i n t}$, (identical for a symmetric barrier) and the exponential decay factor associated with the barrier. The $\mathbf{k}_{\|}$-resolved zero-bias transmission probability at $E_{F}$ evaluated from Eqs. (36) and (37) is plotted in Figs. 8(d)-8(f) for the same values of $\varepsilon^{0}$ of $-3,0$, and $+3 \mathrm{eV}$, respectively. Overall, the agreement between the $\mathbf{k}_{\|}$-resolved current and the $\mathbf{k}_{\|}$-resolved transmission probability is very good.

Since $t=-1 \mathrm{eV}$ in both the leads and the barrier, the exponential factor has its maximum value at $\mathbf{k}_{\|}=0$. On the other hand, according to Eqs. (36) and (37), the projection of the Fermi surface on the BZ allows tunneling of only certain type of interfacial states depending on $\varepsilon^{0}$. These are located around $\mathbf{k}_{\|}=\pi, \pi / 2$, and 0 , for $\varepsilon^{0}=-3,0$, and $+3 \mathrm{eV}$, respectively, i.e., the value of $\left|\mathbf{k}_{\|}^{(\max )}\right|$ at which $T_{\text {int }}$ reaches its maximum value decreases with decreasing (increasing) band fill- 


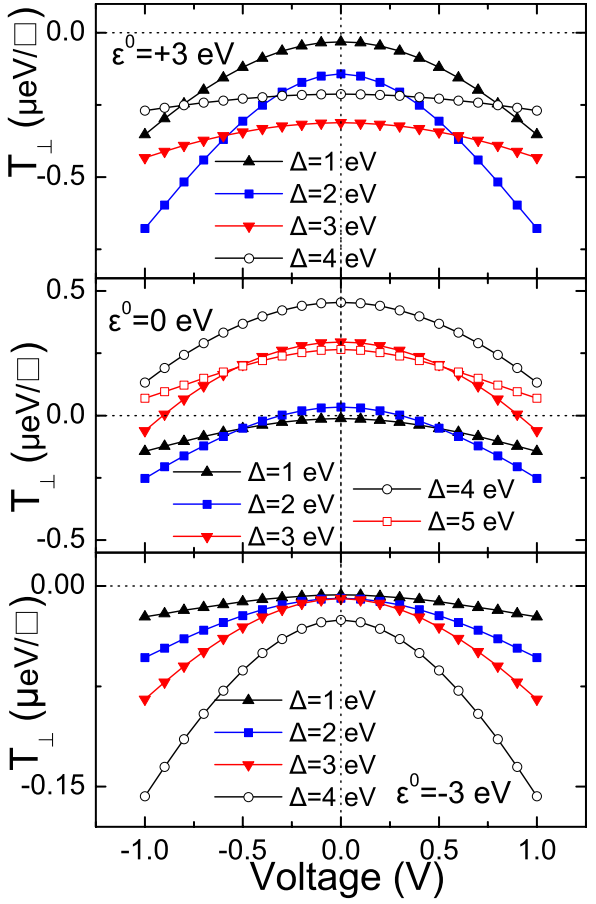

FIG. 9. (Color online) Bias dependence of perpendicular spintransfer torque, $T_{\perp}$, for $\theta=\pi / 2$, various values of the exchange splitting $\Delta$, and three values of the spin-averaged on-site energy $\varepsilon^{0}$, respectively.

ing $\left(\varepsilon^{0}\right)$. Therefore, the most favorable tunneling is for $\varepsilon^{0}=$ $+3 \mathrm{eV}$, since both the exponential factor and $T_{\text {int }}$ are maximum at $\mathbf{k}_{\|}=0$. On the other hand, the density of interfacial states, $N_{i n t}\left(E_{F}\right)$, at $E_{F}$ reaches a maximum at half-filling. Consequently, the maximum value in the total $\mathbf{k}_{\|}$-integrated current as a function of $\varepsilon^{0}$ in Fig. 7 results from the interplay of $N_{\text {int }}\left(E_{F}\right)$, the maximum value of $T_{\text {int }}$ at $\left|\mathbf{k}_{\|}^{(\max )}\right|$, and the maximum of the decaying factor at $\mathbf{k}_{\|}=0$.

\section{B. Voltage dependence of perpendicular spin-transfer torque}

In Fig. 9 we show the bias dependence of the perpendicular component of the net spin torque, $T_{\perp}(V)$, for $\theta=\pi / 2$, various values of the exchange splitting and for $\varepsilon^{0}=+3,0$, and $-3 \mathrm{eV}$. We find that $T_{\perp}(V)$ varies quadratically with bias, as originally suggested, but not calculated, by Slonczewski, ${ }^{23}$ and with $\left(\frac{d^{2} T_{\perp}(V)}{d V^{2}}\right)<0$ for any value of band filling and exchange interaction. ${ }^{24,42}$ These results are consistent with those of recent experiments. ${ }^{32,33}$ Note, that $\left(\frac{d^{2} T_{\perp}(V)}{d V^{2}}\right)$ depends sensitively on both $\Delta$ and the band filling. For example, for $1 / 4$ filling and large exchange splitting $(\Delta$ $=4 \mathrm{eV})\left(\frac{d^{2} T_{\perp}(V)}{d V^{2}}\right) \approx 0$. The zero-bias value of $T_{\perp}(0)$, related to the equilibrium interlayer exchange coupling (IEC), ${ }^{21} \mathrm{de}-$ creases monotonically (nonmonotonically) with increasing $\Delta$ for 3/4 (1/2 and 1/4) filling. At half-filling, $T_{\perp}>0$ for $\Delta$ $\geq 2$ and low bias, and changes sign at larger bias, in contrast to the $1 / 4$ and $3 / 4$ band filling, where $T_{\perp}$ remains negative for any bias. This bias-induced change of $\operatorname{sign}^{42,43}$ of the nonequilibrium IEC may have important practical applications in controlling the sign of the IEC via bias.

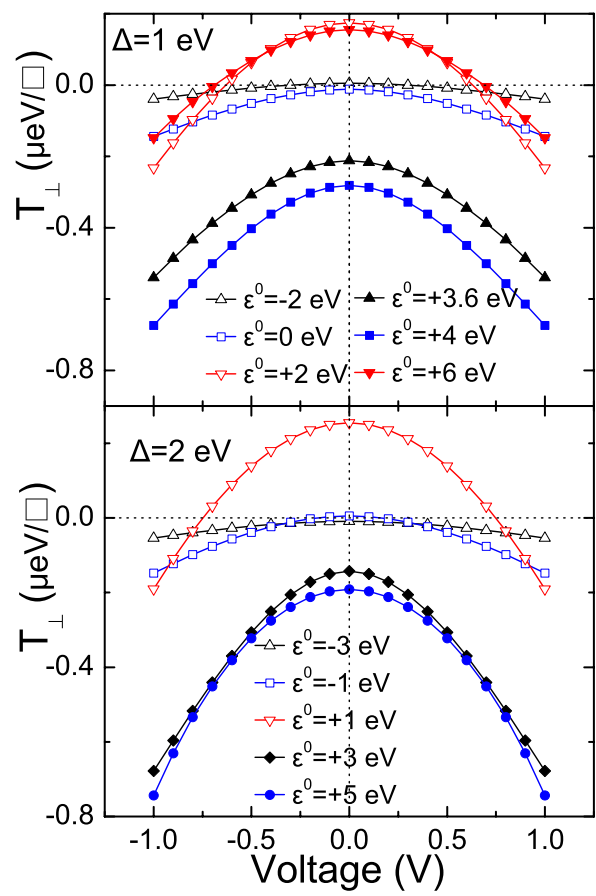

FIG. 10. (Color online) Bias dependence of perpendicular spintransfer torque, $T_{\perp}$, for $\theta=\pi / 2$, various values of the spin-averaged on-site energy $\varepsilon^{0}$, and for $\Delta=1 \mathrm{eV}$ (top panel) and $\Delta=2 \mathrm{eV}$ (bottom panel), respectively.

In Fig. 10 we display the bias dependence of $T_{\perp}$, for $\theta$ $=\pi / 2$, various values of $\varepsilon^{0}$ and for $\Delta=1 \mathrm{eV}$ (top panel) and $\Delta=2 \mathrm{eV}$ (bottom panel), respectively. In all cases we find that $T_{\perp}(V)=T_{\perp}(V=0)-\left|\frac{d^{2} T_{\perp}(V)}{d V^{2}}\right| V^{2}$. Similarly with Fig. 9 we find that the sign of $T_{\perp}(V=0)$ and the bias behavior of the IEC depend on both $\Delta$ and band filling.

\section{Voltage dependence of TMR}

One of the main well-known shortcomings of MRAM is the large decrease in TMR with applied bias voltage. The bias dependence of TMR can be expressed by a characteristic voltage, $V_{1 / 2}$, at which the zero-bias TMR value is halved. A high value of $V_{1 / 2}$ or equivalently a slow decrease in TMR with $V$ is desirable for device applications. ${ }^{10}$ While earlier experiments $^{56}$ found that $V_{1 / 2} \approx 0.2-0.3 \mathrm{~V}$, more recently higher values of $V_{1 / 2}$ of about $0.5-0.6 \mathrm{~V}$ have been reported. ${ }^{57}$

In this section, we present the effect of the TB parameters ( $\Delta$ and $\varepsilon^{0}$ ) on the bias behavior of the TMR. Note that in the half-metallic case, TMR $\rightarrow \infty$ since $I_{\mathrm{AF}} \rightarrow 0$. All figures below show the bias behavior of TMR only for $V>0$ since the TMR is an even function of bias for symmetric MTJs.

In Fig. 11 we show the bias dependence of the TMR for fixed $\varepsilon^{0}=3,0$, and $-3 \mathrm{eV}$ corresponding to $1 / 4$ (top panel), $1 / 2$ (middle panel), and 3/4 band filling (bottom panel), respectively, and for various values of $\Delta$. We find the wellknown result that the TMR decreases with increasing bias. This can be seen more clearly in the insets of Fig. 11, where the TMR normalized to its low-bias value, $\operatorname{TMR}(\mathrm{V}) / \operatorname{TMR}(V \approx 0)$, is plotted versus bias. This is consistent with previous theoretical ${ }^{17,18}$ and experimental ${ }^{9-13}$ re- 


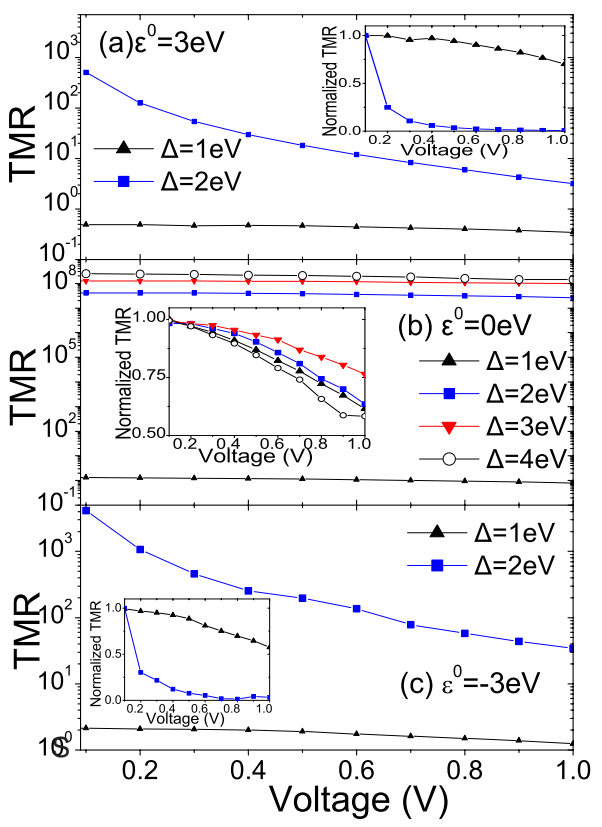

FIG. 11. (Color online) Bias dependence of TMR for various values of exchange splitting $\Delta$ and for three values of the spinaveraged on-site energy, $\varepsilon^{0}$, corresponding to $1 / 4$ (top panel), $1 / 2$ (middle panel), and 3/4 (bottom panel) band filling. Insets display the bias dependence of TMR normalized to its low-bias value, $\operatorname{TMR}(V) / \operatorname{TMR}(V \approx 0)$.

sults. For fixed $\varepsilon^{0}$, the TMR increases (note the logarithmic scale for the TMR) with increasing $\Delta$ due to the decrease in $I(\pi)$, in contrast to the more complex dependence of $T_{\|}$with $\Delta$ shown in Fig. 2. Thus, we find that the TMR and spintransfer torque are not correlated. The TMR increases as $\Delta^{2}$ (exponentially) for small (large) values of $\Delta$. At half-filling $\left(\varepsilon^{0}=0 \mathrm{eV}\right)$, the normalized TMR decreases weakly with bias, independently of the value of $\Delta$ with a high value of $V_{1 / 2} \simeq 1 \mathrm{~V}$, which is about twice the experimental value for clean MTJs. On the other hand, for $1 / 4$ and $3 / 4$ band filling (top and bottom panel insets of Fig. 11), there is a weak (sharp) decrease in the normalized TMR with increasing bias for $\Delta$ of $1 \mathrm{eV}(2 \mathrm{eV})$, where $V_{1 / 2}$ is about $1 \mathrm{~V}(0.2 \mathrm{~V})$, respectively. Thus, these results suggest that for symmetric ideal TMJ without disorder and in the absence of magnon excitations, the $1 / 2$ filling is the optimum value for achieving high values of $V_{1 / 2}$, which are also robust with respect to variations in the exchange splitting.

In Fig. 12 we show the bias dependence of TMR for fixed value of $\Delta$ and different values of $\varepsilon^{0}$, corresponding to different values of band filling. For small exchange splitting, $\Delta=1 \mathrm{eV}$, the TMR is about of the same order of magnitude for any value of $\varepsilon^{0}$ and decreases weakly with bias as can be seen clearly in the inset, thus yielding large values of $V_{1 / 2}$ $\approx 1 \mathrm{eV}$. On the other hand, for $\Delta=2 \mathrm{eV}$, the TMR decreases by several orders of magnitude with increasing (decreasing) $\varepsilon^{0}$ (band filling). Furthermore, the normalized TMR decreases sharply with bias for any $\varepsilon^{0}$, yielding low values for $V_{1 / 2} \approx 0.2 \mathrm{~V}$, in agreement with earlier experiments. ${ }^{56}$ Thus, as for the case of the spin-transfer torque, the TMR and its bias behavior can be selectively tuned with the exchange splitting and the band filling.

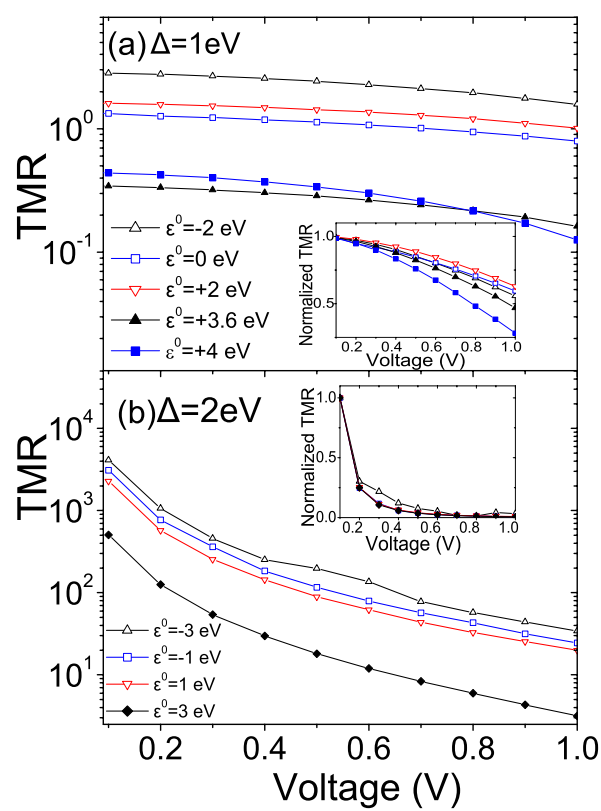

FIG. 12. (Color online) Bias dependence of TMR for various values of the spin-averaged on-site energy $\varepsilon^{0}$ and for two values of exchange splitting of $\Delta=1 \mathrm{eV}$ (top panel) and $\Delta=2 \mathrm{eV}$ (bottom panel), respectively. Insets display the bias dependence of TMR normalized to its low-bias value, $\operatorname{TMR}(V) / \operatorname{TMR}(V \approx 0)$.

\section{CONCLUSION}

In conclusion, we have employed out tight-binding electronic structure calculations and the nonequilibrium Keldysh formalism to study systematically the effect of exchange splitting and band filling on the bias behavior of the fieldlike, $T_{\perp}$, and spin-transfer, $T_{\|}$, components of the spin torque, and the TMR in symmetric MTJ. We find that both components of the spin-transfer torque and the TMR exhibit a wide range of interesting and unusual bias behavior.

We predict a nonmonotonic bias dependence of the spintransfer component of the torque, contrary to the general consensus, where $T_{\|}$may change sign without a sign reversal in bias or current, and it may even have an unexpected quadratic bias dependence. By generalizing the equivalent circuit in Refs. 23 and 24 using angular-dependent resistances, we show that $T_{\|}$satisfies an expression involving the difference in spin currents between the FM and AF configurations. The spin-current density for the FM (AF) alignment is shown to have a linear (quadratic) bias dependence, whose origin lies in the symmetric (asymmetric) nature of the barrier. The interplay of the spin currents for the FM and AF configurations is the key underlying mechanism that leads to a rich behavior of the STT on bias. It should be emphasized that the nonmonotonic bias behavior is not associated with the simple TB model; other systems with more complex electronic structures can also show this behavior, provided that the condition $I_{z}^{S}(0) \approx I_{z}^{S}(\pi)$ is satisfied.

On the other hand, we find that the bias dependence of $T_{\perp}$ is purely quadratic, $T_{\perp}(V)=T_{\perp}(V=0)+\left(\frac{d^{2} T_{\perp}(V)}{d V^{2}}\right) V^{2}$, in the $-1 V \leq V \leq 1 \quad V$ bias range with $d^{2} T_{\perp} / d V^{2}<0$, independent of exchange interaction and band filling. These results are 
consistent with recent experiments. ${ }^{32,33}$ The zero-bias value of the IEC, $T_{\perp}(V=0)$, and $d^{2} T_{\perp} / d V^{2}$ depend both on the exchange splitting and the band filling. Interestingly, at or close to half-filling and for large exchange splitting $T_{\perp}(V)$ changes sign at finite bias, which suggests that the IEC in MTJ may be tuned with bias. The TMR is found to decrease with increasing applied bias voltage but is not correlated with the bias behavior of the spin-transfer torque. The characteristic voltage, $V_{1 / 2}$, at which the zero-bias TMR value is halved can be selectively tuned with the exchange splitting and the band filling. The half-filling is the optimum value for the single-band TB model for achieving high values of $V_{1 / 2}$, which are also robust with respect to variations in the exchange splitting.

\section{ACKNOWLEDGMENTS}

We thank M. Stiles, D. Ralph, and G. Bauer for useful conversations, and J. C. Slonczewski for useful suggestions including the notion that the spin torque can be calculated from the collinear spin currents. The research at California State University Northridge was supported by NSF-PREM under Grant No. DMR-00116566 and by NSF-KITP under Grant No. PHY99-07949. Work at the University of Alabama was supported by NSF MRSEC under Grant No. DMR 0213985 and by the INSIC-EHDR Program and was conducted in part at the Center of Nanophase Materials Sciences, sponsored at Oak Ridge National Laboratory by the Division of Scientific User Facilities, U.S. Department of Energy. *nick.kioussis@csun.edu.

${ }^{1}$ S. Parkin, J. Xin, C. Kaiser, A. Panchula, K. Roche, and M. Samant, Proc. IEEE 91, 661 (2003).

${ }^{2}$ J. Daughton, J. Appl. Phys. 81, 3758 (1997).

${ }^{3}$ M. Hosomi et al., Tech. Dig. - Int. Electron Devices Meet. 2005, 459.

${ }^{4}$ S. Parkin, C. Kaiser, A. Panchula, P. Rice, B. Hughes, M. Samant, and S.-H. Yang, Nature Mater. 3, 862 (2004).

${ }^{5}$ S. Yuasa, T. Nagahama, Y. Suzuki, and K. Ando, Nature Mater. 3, 868 (2004).

${ }^{6}$ Y. Lee, J. Hayakawa, S. Ikeda, F. Matsukura, and H. Ohno, Appl. Phys. Lett. 90, 212507 (2007).

${ }^{7}$ W. H. Butler, X. G. Zhang, T. C. Schulthess, and J. M. MacLaren, Phys. Rev. B 63, 054416 (2001).

${ }^{8}$ J. Mathon and A. Umerski, Phys. Rev. B 63, 220403(R) (2001).

${ }^{9}$ J. Hayakawa, K. Ito, S. Kokado, M. Ichimura, A. Sakuma, M. Sugiyama, H. Asano, and M. Matsui, J. Appl. Phys. 91, 8792 (2002).

${ }^{10}$ D. Wang, C. Nordman, J. M. Daughton, Z. Qian, and J. Fink, IEEE Trans. Magn. 40, 2269 (2004).

${ }^{11}$ H. Boeve, E. Girgis, J. Schelten, J. D. Boeck, and G. Borghs, Appl. Phys. Lett. 76, 1048 (2000).

${ }^{12}$ M. Sharma, S. X. Wang, and J. H. Nickel, Phys. Rev. Lett. 82, 616 (1999)

${ }^{13}$ S. O. Valenzuela, D. J. Monsma, C. M. Marcus, V. Narayanamurti, and M. Tinkham, Phys. Rev. Lett. 94, 196601 (2005).

${ }^{14}$ C. Tiusan, J. Faure-Vincent, C. Bellouard, M. Hehn, E. Jouguelet, and A. Schuhl, Phys. Rev. Lett. 93, 106602 (2004).

${ }^{15}$ S. Zhang, P. M. Levy, A. C. Marley, and S. S. P. Parkin, Phys. Rev. Lett. 79, 3744 (1997).

${ }^{16}$ J. S. Moodera, J. Nowak, and R. J. M. van de Veerdonk, Phys. Rev. Lett. 80, 2941 (1998).

${ }^{17}$ J. Zhang and R. M. White, J. Appl. Phys. 83, 6512 (1998).

${ }^{18}$ F. fei Li, Z.-zhong Li, M.-wen Xiao, J. Du, W. Xu, A. Hu, and J. Q. Xiao, J. Appl. Phys. 95, 7243 (2004).

${ }^{19}$ C. Heiliger, P. Zahn, B. Y. Yavorsky, and I. Mertig, Phys. Rev. B 72, 180406(R) (2005).

${ }^{20}$ C. Heiliger, P. Zahn, and I. Mertig, J. Magn. Magn. Mater. 316, 478 (2007)

${ }^{21}$ J. C. Slonczewski, Phys. Rev. B 39, 6995 (1989).
${ }^{22}$ L. Berger, Phys. Rev. B 54, 9353 (1996).

${ }^{23}$ J. C. Slonczewski, Phys. Rev. B 71, 024411 (2005).

${ }^{24}$ I. Theodonis, N. Kioussis, A. Kalitsov, M. Chshiev, and W. H. Butler, Phys. Rev. Lett. 97, 237205 (2006).

${ }^{25}$ P. M. Levy and A. Fert, Phys. Rev. Lett. 97, 097205 (2006).

${ }^{26}$ M. Wilczynski, J. Barnas, and R. Swirkowicz, Phys. Rev. B 77, 054434 (2008).

${ }^{27}$ J. Xiao, G. E. W. Bauer, and A. Brataas, Phys. Rev. B 77, 224419 (2008).

${ }^{28}$ C. Heiliger and M. D. Stiles, Phys. Rev. Lett. 100, 186805 (2008).

${ }^{29}$ A. Manchon, N. Ryzhanova, A. Vedyayev, M. Chschiev, and B. Dieny, J. Phys.: Condens. Matter 20, 145208 (2008).

${ }^{30}$ M. Tsoi, A. Jansen, J. Bass, W.-C. Chiang, M. Seck, V. Tsoi, and P. Wyder, Phys. Rev. Lett. 81, 493 (1998).

${ }^{31}$ J. A. Katine, F. J. Albert, R. A. Buhrman, E. B. Myers, and D. C. Ralph, Phys. Rev. Lett. 84, 3149 (2000).

${ }^{32}$ J. C. Sankey, Y.-T. Cui, J. Z. Sun, J. Slonczweski, R. A. Buhrman, and D. C. Ralph, Nat. Phys. 4, 67 (2008).

${ }^{33}$ H. Kubota, et al., Nat. Phys. 4, 37 (2008).

${ }^{34}$ S. Petit, C. Baraduc, C. Thirion, U. Ebels, Y. Liu, M. Li, P. Wang, and B. Dieny, Phys. Rev. Lett. 98, 077203 (2007).

${ }^{35}$ Z. Li, S. Zhang, Z. Diao, Y. Ding, X. Tang, D. M. Apalkov, Z. Yang, K. Kawabata, and Y. Huai, Phys. Rev. Lett. 100, 246602 (2008).

${ }^{36}$ Y. Huai, F. Albert, P. Nguyen, M. Pakala, and T. Valet, Appl. Phys. Lett. 84, 3118 (2004).

${ }^{37}$ G. Fuchs, N. Emley, I. Krivorotov, P. Braganca, E. Ryan, S. Kiselev, J. Sankey, D. Ralph, R. Buhrman, and J. Katine, Appl. Phys. Lett. 85, 1205 (2004).

${ }^{38}$ S. Kiselev, J. C. Sankey, I. N. Krivorotov, N. C. Emley, R. J. Schoelkopf, R. A. Buhrman, and D. C. Ralph, Nature (London) 425, 380 (2003).

${ }^{39}$ S. Kaka, M. R. Pufall, W. H. Rippard, T. J. Silva, S. E. Russek, and J. A. Katine, Nature (London) 437, 389 (2005).

${ }^{40}$ F. Mancoff, N. D. Rizzo, B. N. Engel, and S. Tehrani, Nature (London) 437, 393 (2005).

${ }^{41}$ J. Katine and E. F. Fullerton, J. Magn. Magn. Mater. 320, 1217 (2008).

${ }^{42}$ M. Chshiev, I. Theodonis, A. Kalitsov, N. Kioussis, and W. H. 
Butler, IEEE Trans. Magn. 44, 2543 (2008).

${ }^{43}$ P. M. Haney, C. Heiliger, and M. D. Stiles, Phys. Rev. B 79, 054405 (2009).

${ }^{44}$ C. Caroli, R. Combescot, P. Nozieres, and D. Saint-James, J. Phys. C 4, 916 (1971).

${ }^{45}$ E. M. Lifshitz and L. P. Pitaevskii, Physical Kinetics, Course of Theoretical Physics (Pergamon, Oxford, 1981), Vol. 10.

${ }^{46}$ M. D. Stiles and A. Zangwill, Phys. Rev. B 66, 014407 (2002).

${ }^{47}$ D. M. Edwards, F. Federici, J. Mathon, and A. Umerski, Phys. Rev. B 71, 054407 (2005).

${ }^{48}$ A. Brataas, G. E. Bauer, and P. J. Kelly, Phys. Rep. 427, 157 (2006).

${ }^{49}$ A. Kalitsov, I. Theodonis, N. Kioussis, M. Chshiev, W. H. Butler, and A. Vedyayev, J. Appl. Phys. 99, 08 G501 (2006).

${ }^{50}$ M. Stamenova, S. Sanvito, and T. N. Todorov, Phys. Rev. B 72, 134407 (2005).
${ }^{51}$ S. Zhang and Z. Li, Phys. Rev. Lett. 93, 127204 (2004).

${ }^{52}$ A. Deac, H. K. Akio Fukushima, H. Maehara, Y. Suzuki, S. Yuasa, Y. Nagamine, K. Tsunekawa, D. D. Djayaprawira, and N. Watanabe, Nat. Phys. 4, 803 (2008).

${ }^{53}$ W. F. Brinkman, R. C. Dynes, and J. M. Rowell, J. Appl. Phys. 41, 1915 (1970).

${ }^{54}$ K. D. Belashchenko, E. Y. Tsymbal, M. van Schilfgaarde, D. A. Stewart, I. I. Oleinik, and S. S. Jaswal, Phys. Rev. B 69, 174408 (2004).

${ }^{55}$ X.-G. Zhang and W. H. Butler, J. Phys. Condens. Matter 15, R1603 (2003).

${ }^{56}$ J. S. Moodera, L. R. Kinder, T. M. Wong, and R. Meservey, Phys. Rev. Lett. 74, 3273 (1995).

${ }^{57}$ S.-J. Ahn, T. Kato, H. Kubota, Y. Ando, and T. Miyazaki, Appl. Phys. Lett. 86, 102506 (2005). 